Abstract

\title{
Generalized Inference for the Efficient Reconstruction of Weighted Networks ${ }^{\dagger}$
}

\author{
Federica Parisi ${ }^{1}$, Tiziano Squartini ${ }^{2}$ and Diego Garlaschelli ${ }^{2}$ \\ 1 ViriCiti, Amsterdam, The Netherlands \\ 2 IMT School for Advanced Studies Lucca, Lucca, Italy \\ + Presented at the Entropy 2021: The Scientific Tool of the 21st Century, 5-7 May 2021; Available online: \\ https://sciforum.net/conference/Entropy2021/.
}

Published: 5 May 2021

Introduction. Network reconstruction is an active field of research. Among the methods proposed so far, some assume that the binary and weighted constraints jointly determine the reconstruction output; others consider the weights estimation step as completely unrelated to the binary one. Amidst the former ones, a special mention is deserved by the Enhanced Configuration Model; the algorithms of the second group, instead, are those iteratively adjusting the link weights on top of some previouslydetermined topology.

Methods and Results. Here we develop a theoretical framework that provides an analytical, unbiased procedure to estimate the weighted structure of a network, once its topology has been determined, thus extending the Exponential Random Graph (ERG) recipe. Our approach treats the information about the topological structure as a priori; together with the proper weighted constraints, it represents the input of our generalized reconstruction procedure. The probability distribution describing link weights is, then, determined by maximizing the key quantity of our algorithm, i.e., the conditional entropy under a properly-defined set of constraints. This algorithm returns a conditional probability distribution depending on a vector of unknown parameters; in alignment with previous results, their estimation is carried out by considering a generalized likelihood function. In our work, we compare two possible specifications of this framework.

Conclusions. The knowledge of the structure of a financial network gives valuable information for estimating the systemic risk. However, since financial data are typically subject to confidentiality, network reconstruction techniques become necessary to infer both the presence of connections and their intensity. Recently, several "horse races" have been conducted to compare the performance of these methods. Here, we establish a generalised likelihood approach to rigorously define and compare methods for reconstructing weighted networks: the best one is obtained by "dressing" the bestperforming, available binary method with an exponential distribution of weights.

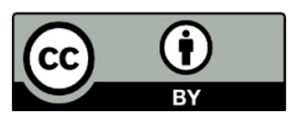

(C) 2021 by the author. Licensee MDPI, Basel, Switzerland. This article is an open access article distributed under the terms and conditions of the Creative Commons Attribution (CC BY) license (http://creativecommons.org/licenses/by/4.0/). 